\title{
Optical and microwave observation of forest restoration after abnormal fires
}

\author{
Aleksey V. Dmitriev ${ }^{1}$, Tumen N. Chimitdorzhiev ${ }^{1}$, Pavel N. Dagurov ${ }^{1}$ and \\ Irina I. Kirbizhekova ${ }^{1}$ \\ ${ }^{1}$ Institute of Physical Materials Science, SB RAS, Ulan-Ude, Russia
}

\begin{abstract}
The paper discusses the possibility of a forest undergrowth detecting with help of polarimetric decompositions and time series analysis of ALOS-2/PALSAR-2 and Sentinel-1 radar images. The presented results show principal possibility of confidently identifying the growth of forest stands.
\end{abstract}

Keywords

Reforestation, polarimetric decompositions, time-series analysis, ALOS-2/PALSAR-2, Sentinel-1/2.

\section{Introduction}

Modern climate changes are largely associated with the problem of carbon balance, the main source of which is tropical and boreal forests. Deforestation and wildfires have significantly reduced the area of the forest. Russian Federation has a federal project named "Forest Conservation" currently, the purpose of which is reforestation. Territories suitable for reforestation are preliminarily identified using data from multispectral optical sensors within the framework of this project. Next, field observations and soil analysis are performed for further planting of seedlings. However, due to the specifics of optical images, territories for planting forest seedlings are often mistakenly allocated, which, as a result of self-seeding, are already covered with forest. Identification of the natural restoration of the forest is difficult due to low-growing young deciduous and rare coniferous trees are poorly distinguishable against the background of dense grass and shrubs. This report examines the possibility of identifying undergrowth of forest stands by analyzing multitemporal polarimetric radar images of ALOS-2 PALSAR-2. For comparison, the results obtained from the time series of the Sentinel-1 C-band radar with dual polarization are also presented.

\section{Study area description}

Study area is located at Eastern Siberia, Russia, east of the Lake Baikal in central part of Buryatia Republic (see Figure 1). It is a mountainous hard-to-reach region covered by different types of forest. A strong wildfire in the summer of 2007 destroyed the forest on a large territory of this site. Four different sites were selected for detailed study within study area, which are

SDM-2021: All-Russian conference, August 24-27, 2021, Novosibirsk, Russia

$\bigcirc$ dmitriev.aleksey@gmail.com (A.V. Dmitriev)

(c) (1) $\odot 2021$ Copyright for this paper by its authors. Use permitted under Creative Commons License Attribution 4.0 International (CC BY 4.0).

CEUR Workshop Proceedings (CEUR-WS.org) 
a

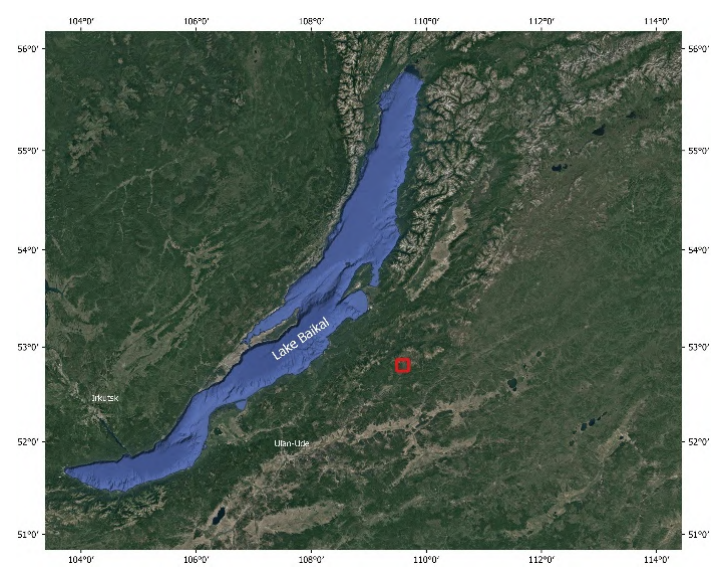

b

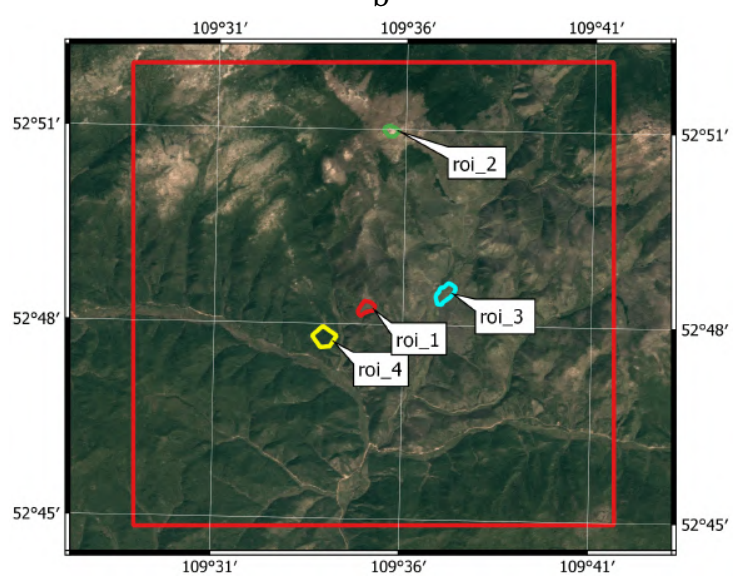

Figure 1: Study area on the Google Maps and locations of the regions of interest (roi).

a

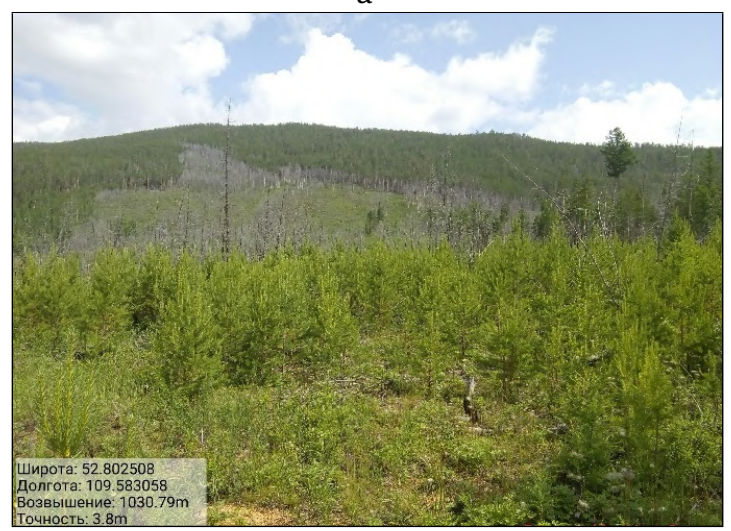

b



Figure 2: Pictures of roi_1 (a) and roi_3 (b).

shown in the Figure 1, b as roi_1-roi_4. roi_1 is the overgrown territory of forest after a fire with approximate area equaled to $12 \mathrm{Ha}$ (see Figure 2, a). roi_2 is the rocky treeless site. Before abnormal wildfire it was partially covered by cedar trees which were completely destroyed by the strong fire. roi_3 represents wetlands with shrubs located in floodplain of the river (see Figure 2, b). roi_4 is the mature pine forest not affected by wildfires.

Areas of burned forest are usually surrounded by surviving forest, due to which natural reforestation takes place. Also, small groups of surviving mature trees are natural sources of seeds in the burned areas.

\section{Research methodology and data used}

Several different methods were used to identify the undergrowth of forest stands. 
1. Analysis of multitemporal polarimetric decompositions that distinguish the dominant scattering mechanisms. For example, volume scattering from the forest stands, surface from the rough surface, double-bounce from natural targets. Since the forest vegetation is destroyed after abnormal wildfires, it is assumed that there is a double-bounce scattering (for example, scattering from "dihedral" reflector formed by the earth's surface and the remains of a tree trunk) and a surface scattering. After self-seeding and the growth of new trees, it is assumed that the volume scattering component will increase and the surface and double-bounce scattering components will decrease. Model-based decomposition, proposed by Freeman and Durden [1] and Claude - Pottier eigenvalues/eigenvectors decomposition [2] were used for analysis.

2. Analysis of temporal variations of radar backscattering amplitude and interferometric coherence at different polarizations and in different bands. It is expected that the growth of forest stands will lead to an increase in the amplitude of the radar signal due to an increase in the effective "roughness" of the reflecting surface. At the same time, there should be a decrease in the interferometric coherence.

3. Normalized difference vegetation index (NDVI) time series analysis of Sentinel-2A/B satellites to obtain dynamics of overgrowing forestless areas. The analysis was made with help of Google Earth Engine cloud platform.

We used data of Japanese L-band synthetic aperture radar (SAR) PALSAR-2 aboard the ALOS2 satellite which was launched in May, 2014. ALOS-2/PALSAR-2 has different observation modes including two modes with full (quad) polarization. Unfortunately, only two fully polarimetric scenes were available for the study area. Therefore, seven images with dual polarization $(\mathrm{HH} / \mathrm{HV})$ were selected for processing (see Table 1$)$.

Also, we used data of Sentinel-1B SAR. Sentinel-1B is a European radar imaging satellite launched on 25 April 2016. It is the second of two satellites in the Sentinel-1 constellation. The satellite carries a C-band sensor (wavelength is equal $5.6 \mathrm{~cm}$ ) operating in four exclusive imaging modes with different resolution and coverage. It provides dual polarization capability $(\mathrm{VV} / \mathrm{VH})$ and 12-days revisit time. We chose data imaged from relative orbit 62 for regions of interest. 111 IW SLC images from February 25, 2017 to February 28, 2021 were processed totally.

Sentinel-2 optical multispectral images were used to create multitemporal NDVI time series for test sites. The Sentinel-2 mission is a constellation with two twin satellites, Sentinel-2A

Table 1

ALOS-2/PALSAR-2 data.

\begin{tabular}{ccc}
\hline Mode & HBQ (Quad pol.) & FBD (Dual pol.) \\
\hline \multirow{4}{*}{ Scene IDs } & ALOS2159621050-170507 & ALOS2116861050-140928 \\
& & ALOS2050-160717 \\
& ALOS2283821050-190825 & ALOS2223791050-180715 \\
& & ALOS2277611050-190714 \\
& & ALOS2331431050-200712 \\
\hline
\end{tabular}


(launched on June 23, 2015) and Sentinel-2B (launched on March 7, 2017). The satellites each carry a single multi-spectral instrument (MSI) with 13 spectral channels in the visible/near infrared (VNIR) and short wave infrared spectral range (SWIR) for surveying with a resolution of 10 to $60 \mathrm{~m}$. The presence of two satellites in the mission allow repeated surveys every 5 days. More than one hundred cloudless images were used for NDVI time series calculation.

All SAR's data were processed in ESA SNAP 8.0, free and open source software [3]. Calculation of vegetation index was performed with help of Google Earth Engine cloud platform [4].

\section{Results and discussion}

\subsection{ALOS-2/PALSAR-2}

\subsubsection{Fully polarimetric data}

Let us consider polarimetric decomposition, proposed by Freeman and Durden [1]. It is based on the three-component scattering mechanism model. The span $\mathrm{P}$ of radar return is assumed to be the sum of three independent terms: the contribution of a single (surface), double-bounce (or even-bounce) and a volume (multiple) scattering components:

$$
P=\left|S_{H H}\right|^{2}+2\left|S_{H V}\right|^{2}+\left|S_{V V}\right|^{2}=P_{s}+P_{d}+P_{v},
$$

where $s, d$ and $v$ indexes denote single, double-bounce and volume scattering respectively. Surface (single) scattering is a typical mechanism of scattering from soil or water. Doublebounce scattering occurs when there are vertically oriented targets on a horizontal surface and is often found in built-up areas (reflections from the ground and walls), as well as in forests (reflections from trunks and soil). Canopy (volume) scattering occurs where there is a cloud of randomly oriented, very thin, cylinder-like scatterers - for example, tree branches.

The Table 2 shows the results of three-component Freeman - Durden decomposition applied to fully polarimetric ALOS-2/PALSAR-2 data. The values in the table are expressed as a percentage. First value in cells corresponds to May 7, 2017 (ALOS2159621050-170507), second one to May 25, 2019 (ALOS2283821050-190825). All results are mean values calculated for respective roi.

It is evident, that volume scattering increased at the sites roi_1, roi_2, roi_3. The maximum increase about $20 \%$ is observed for overgrown area of forest after fire (roi_1) and rocky treeless area (roi_2). In the first case, the forest undergrowth grew up by $0.5-1.5 \mathrm{~m}$ over two years with a corresponding increase in the total number of scatterers, such as tree trunks and branches. The increased forest biomass leads to surface "screening" effect resulting in decreased surface

Table 2

Results of three-component Freeman - Durden decomposition.

\begin{tabular}{ccccc}
\hline & roi_1 & roi_2 & roi_3 & roi_4 \\
\hline$P_{d}, \%$ & $5.9 / 5.3$ & $5.4 / 4.5$ & $5 / 7.9$ & $7.6 / 6.2$ \\
$P_{s}, \%$ & $49.2 / 29$ & $52 / 33.5$ & $66 / 49.8$ & $7.3 / 8.8$ \\
$P_{v}, \%$ & $44.9 / 65.7$ & $42.6 / 62$ & $29 / 42.3$ & $85.1 / 85$ \\
\hline
\end{tabular}


scattering component by the same $20 \%$. Growth of volume scattering component at roi_3 mainly associated with shrubs density increasing. The scattering components variations are not significant for the mature forest.

\subsubsection{Dual polarimetric data}

Unfortunately, due to small amount of fully polarimetric data (only 2 scenes) we can't analyze dynamics of overgrowing. But there are more images made in FBD mode $(\mathrm{HH} / \mathrm{HV}$ polarizations) which we can analyze (see Table 1).

For dual polarimetric data we can only perform Cloude - Pottier decomposition [3] which is based on the analysis of the coherency matrix eigenvalues ( $H \alpha$ decomposition). The $H$ parameter called entropy, describes the randomness degree of scattering. High entropy values usually indicate the presence of vegetation. The angle $\alpha$ indicates the radar backscattering mechanism.

Figure 3 shows entropy and $\alpha$ time series calculated from the data of ALOS-2/PALSAR-2 in FBD observation mode. The data obtained was averaged within the test site to get one value per roi for each date.

The plots in Figure 3 show that neither the entropy nor the $\alpha$ parameter estimated from dual polarization data underwent changes significantly over 5 years. So, they cannot serve as the indicators of the reforestation process.

\subsubsection{Amplitude and coherence time series}

Since the polarimetric decomposition of the dual polarimetric data did not reveal considerable changes in the test areas, it was decided to analyze other characteristics of the scattered signal such as amplitude and coherence. Figure 4 shows the temporal dependencies of radiometrically calibrated and normalized amplitude and sequential interferometric coherence (first date with second, second with third etc.) for different polarization states.

The amplitude plots show that it is almost impossible to distinguish forest undergrowth from other areas. For example, the correlation between the plot for roi_1 (forest undergrowth) and the plot for roi_2 (rocky treeless area) is 0.92 for $\mathrm{HH}$ polarization and 0.96 for HV. The only area that can be identified by the amplitude plots is the mature forest (roi_4). The radar signal at cross-polarization is on average $4.5 \mathrm{~dB}$ higher than at other sites due to scattering on large tree branches. The situation is similar in terms of coherence. The sequential coherence on roi_1 has

a

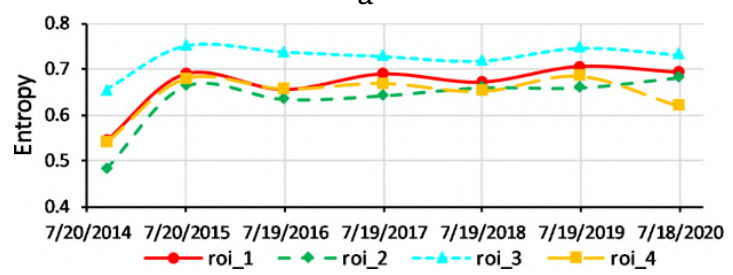

Figure 3: Temporal dependencies of entropy of ALOS-2/PALSAR-2. b

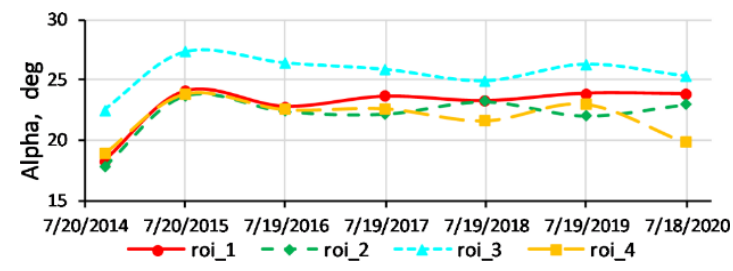

(a) and a angle (b) for dual polarization data 

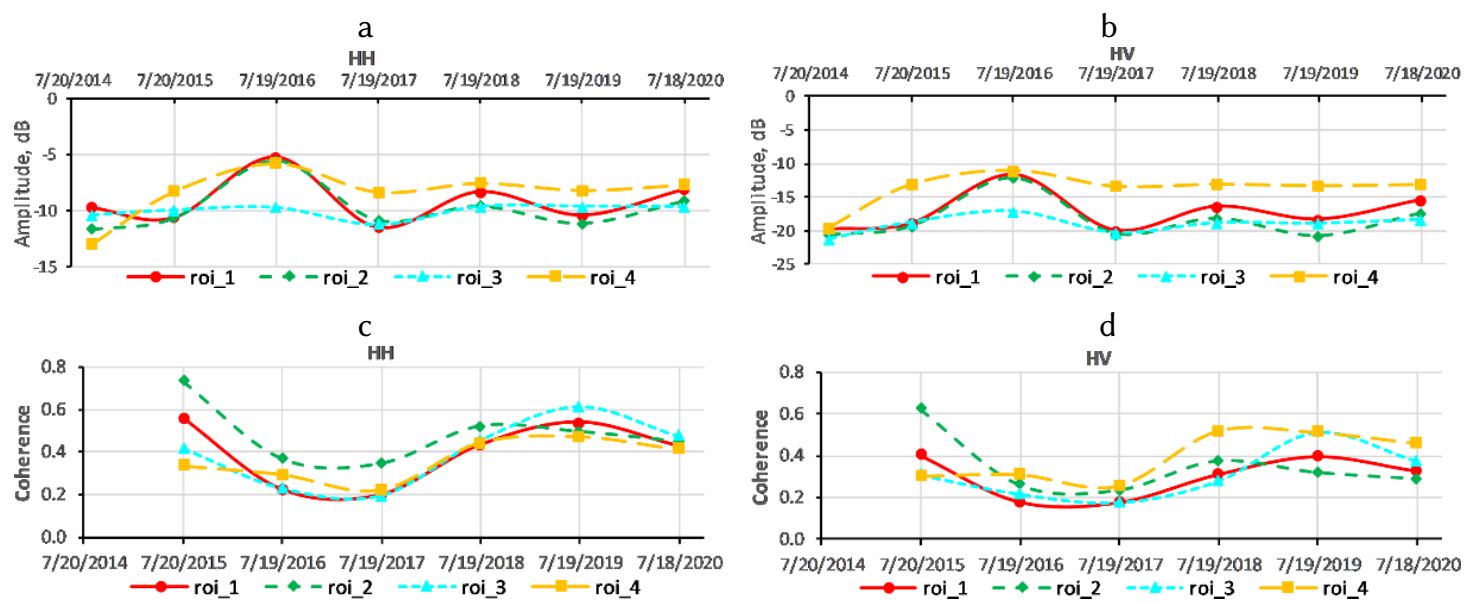

Figure 4: Temporal dependencies of radiometrically calibrated and normalized amplitude (a, b) and sequential coherence $(c, d)$.

a significant positive correlation (greater than 0.55 ) with all other sites. Thus, the L-band data with dual polarization is not suitable for monitoring the reforestation process. This is mainly due to the high penetration of L-band waves. A low growing forest does not attenuate the radar backscattering significantly at a time interval of 7 years, even despite its relative density nor does it lead to visible depolarization effects.

\subsection{Sentinel-1B}

Due to the inability to reveal the forest restoration in the L-band, it was decided to use the freely available data of the European Sentinel-1B all-weather radar operating in C-band (wavelength $5.6 \mathrm{~cm}$ ). Sentinel-1B survey frequency for the territory of Siberia is 12 days. Regular survey of the study area has been conducted since 2017 . Sentinel-1 radar emits vertically polarized waves (VV and $\mathrm{VH}$ ) unlike the ALOS-2 PALSAR-2, which mainly operates at horizontal polarization on radiation $(\mathrm{HH}$ and $\mathrm{HV})$.

\subsection{1. $H / \alpha$ decomposition}

Figure 5 shows the calculated time series of entropy and $\alpha$ angle for each roi.

a

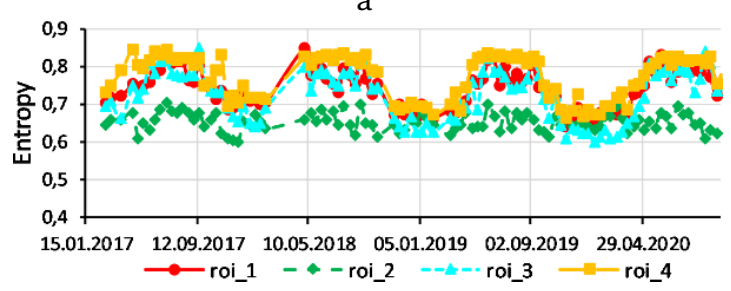

b

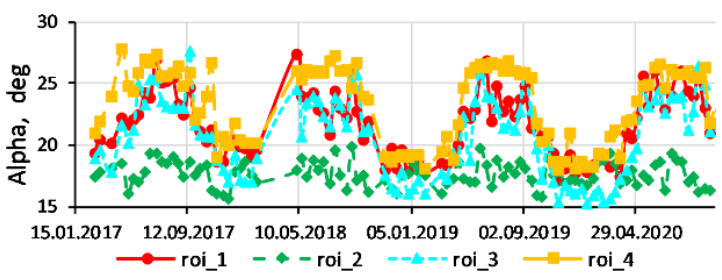

Figure 5: Temporal dependencies of entropy (a) and $\alpha$ angle (b) for Sentinel-1B data. 
In this case, the $H / \alpha$ polarimetric decomposition allows us to separate areas with vegetation from a treeless area by the entropy value and the scattering mechanism. However, as before, the vegetation areas are not divided among themselves. It should also be noted that there is a distinct seasonal dependence of both the entropy and the $\alpha$ angle.

\subsubsection{Amplitude and coherence time series}

Let us consider temporal dependencies of radiometrically calibrated and normalized backscattering amplitude. Due to the smaller wavelength in C-band the radar backscattering is very sensitive to changes in the underlying media, for example, in variations of soil moisture, freezing/thawing processes, etc. All these factors strongly influence to the magnitude of the amplitude, which is especially noticeable in the summer and autumn-spring periods (see the seasonal dependence in Figure 5), and are expressed in a large variance of the measurement results. Therefore, to assess the reforestation processes, it was decided to consider more stable winter data. A total of 38 winter images were used, taken between the beginning of November of one year until the end of February of the year following it. The obtained results are presented in Figure 6. The plots in Figure 6, a for the vertical co-polarization shows continuous increasing of the backscattered signal for roi_1 and roi_3. A comparison of the average amplitude value for the winter period of 2017-2018 with the same period of 2020-2021 shows it growth up by $11 \%$ and $12 \%$ for roi_1 and roi_3 respectively.

It clearly visible that the amplitude trend lines are almost parallel for these areas. It indicates similar scattering processes. The radar backscattering also increases for roi_2 and roi_4, but it is significantly less in quantitative terms ( $<2 \%$ for roi_2 and $6 \%$ for roi_4). The dependencies for cross-polarization (Figure 6, b) show a similar trend for roi_1 and roi_3, but are less expressed
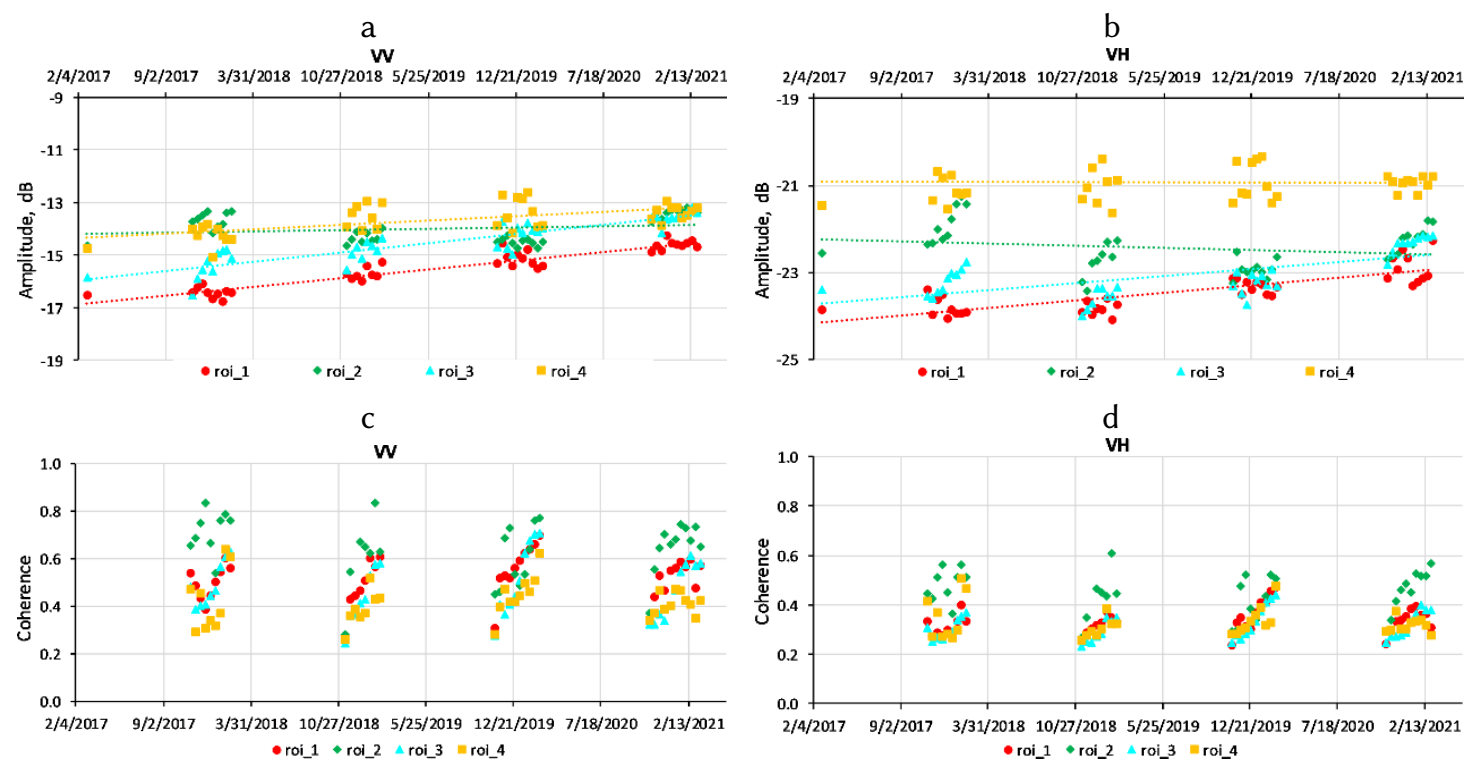

Figure 6: Temporal dependencies of radiometrically calibrated and normalized amplitude (a, b) and sequential coherence $(c, d)$ during winter. 
quantitatively ( $<4 \%$ for both sites). roi_4 should be noted individually because it demonstrates the stability of the average reflective properties over 4 years (variations are equal to $-0.6 \%$ ). The behavior of radar backscattering on roi_2 is decreasing in contrary to co-polarization case. It is interesting to compare roi_2 and roi_3 within the same winter season. As can be clearly seen for the seasons 2017/2018, 2018/2019 and 2020/2021, the radar backscattering consistently increases during the winter. Obviously, this is due to snow cover growth (which can exceed $1 \mathrm{~m}$ in a given area) and an increase in the volume scattering component in the total reflected signal. However, if roi_2 is a rocky treeless area, then roi_3 is the shrubs in the floodplain of river. So, we can conclude that in winter, when the shrub sheds its foliage and the soil freezes, the backscattering from the site with deciduous undergrowth becomes similar to the backscattering from a treeless site, i.e. deciduous young growth becomes "radio-transparent". Coniferous forest undergrowth on roi_1 does not show such behavior. This can serve as an indirect indicator for the classification of forest undergrowth by vegetation type.

Figures 6, $\mathrm{c}$ and $\mathrm{d}$ show the coherence time series. The highest coherence values are observed in the treeless area as expected. However, no unambiguous conclusions can be drawn from these plots for the rest of the forest sites.

Thus, the analysis of the temporal series of radiometrically calibrated and normalized backscattering amplitude in C-band allow us to reliably detect forest undergrowth of both coniferous and deciduous species and distinguish it from mature forest and treeless areas. Forest undergrowth can also be divided by species composition with help of indirect features.

We must point out that all these conclusions are true for the low height undergrowth only. If the trends shown in Figures 6, a and b will proceed, then saturation effect will occur in 5-6 years, when the coniferous undergrowth reaches a height of 2.5-3 $\mathrm{m}$ and the undergrowth will be impossible to distinguish from the mature forest. However, the increased forest biomass should affect the backscattering in L-band in this case.

\subsection{Sentinel-2A/B}

As an additional step we investigated normalized difference vegetation index (NDVI) obtained from Sentinel-2A/B satellites data. Calculation of NDVI was performed with help of Google Earth Engine cloud platform. More than one hundred cloudless images were used for NDVI time series calculation. (see Figure 7).

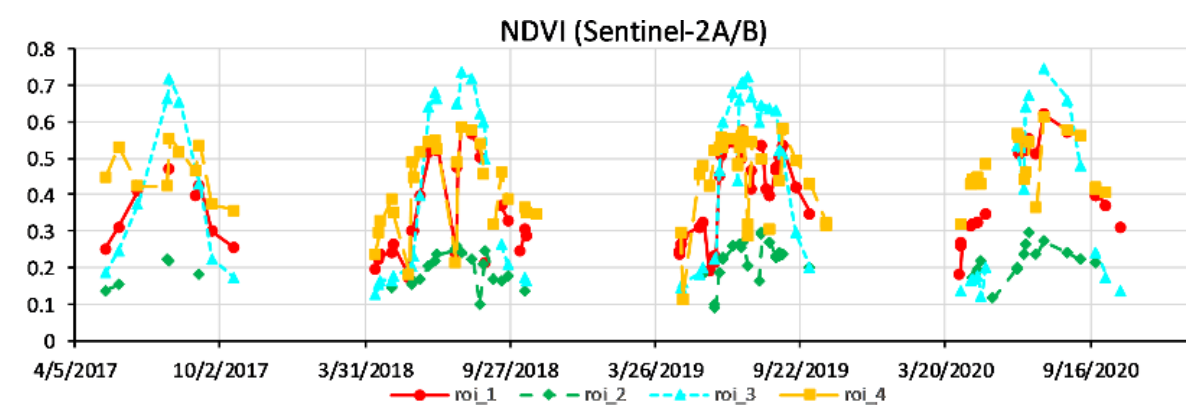

Figure 7: Temporal dependencies of NDVI. 
It is evident that NDVI can't identify young growth. But it can help separate different types of undergrowth. For example, shrubs at roi_3 have a higher value of NDVI than young growth at roi_1. Rocky treeless area (roi_2) has NDVI less than 0.3 during summer period. It means that increased volume scattering, as was shown by polarimetric decomposition (see Table 2), not essentially related with vegetation. Vegetation index at roi_1 almost follows NDVI values at roi_4, where pine forest is located. So, we can conclude that undergrowth at roi_1 contains predominantly young pines.

\section{Conclusion}

The paper discusses the possibility of a forest undergrowth detecting with help of time series analysis of ALOS-2/PALSAR-2 and Sentinel-1 radar images. It is shown that model-based decompositions of fully polarimetric data in L-band (ALOS-2/PALSAR-2) allow us to confidently identify the growth of forest stands. However, data with double polarization are no longer able to separate forest undergrowth from other types of vegetation. On the contrary, the C-band (Sentinel-1) data with a vertical co-polarization taken in the winter period make it possible to determine the forest undergrowth by virtue of time series analysis of radiometrically calibrated and normalized amplitude. The use of optical sensors data, in particular Sentinel-2A/B, allows us to classify the identified undergrowth by species composition and eliminate classification errors in treeless areas, which show increased volume scattering on model-based polarimetric decompositions.

\section{Acknowledgments}

The authors would like to thank Japanese aerospace agency (JAXA) for ALOS-2/PALSAR-2 data granted under ALOS-2 RA6 (PI-3092). The research was carried out within the state assignment of Ministry of Science and Higher Education of the Russian Federation and partially supported by the Russian Foundation for Basic Research (grant No. 18-47-030001-r_a).

\section{References}

[1] Freeman A., Durden S.L. A three-component scattering model for polarimetric SAR data // IEEE Trans. Geosci. Remote Sens. 1998. Vol. 36. No. 3. P. 963-973.

[2] Cloude S.R., Pottier E. An entropy based classification scheme for land applications of polarimetric SAR // IEEE Trans. Geosci. Remote Sens. 1997. Vol. 35. P. 68-78.

[3] ESA SNAP. URL: https://step.esa.int (accessed June 23, 2021).

[4] Google Earth Engine. URL: https://earthengine.google.com (accessed June 23, 2021). 